\title{
Quality of Service Assurances in Multimedia Network: A Simulation Approach
}

\author{
Suartana I Made $^{1, *}$, and Prapanca Aditya ${ }^{1}$ \\ ${ }^{1}$ Universitas Negeri Surabaya, Fakultas Teknik, 60231 Ketintang Surabaya, Indonesia
}

\begin{abstract}
QoS is not a consideration at the beginning of the development of IP networks. However, as its growth and support more services including critical and real-time services, QoS support is indispensable. Multimedia applications require different QoS requirements from simple network applications. Multimedia services need more bandwidth, lossless delivery, and strict delay. IP networks treat all traffic, in the same way, this will be a problem for real-time traffic likes multimedia applications. This project uses MultiProtocol Label Switching (MPLS) and DiffServ QoS management schemes to meet the quality of service requirement in multimedia networks. The aim of this study shows that two methods can improve network performance for multimedia type applications. An analysis to compare the quality of service guarantees provided by these models using a simulation approach. Based on simulation results and analysis shows that two methods improved network performance for multimedia type applications in massive traffic environments.
\end{abstract}

\section{Introduction}

The Quality of Service (QoS) support for real-time applications has increased rapidly. Unfortunately, the current IP network only support best-effort services. Internet does not provide QoS guarantee to support such a real-time application [1].

With the increase, new multimedia internet services like Voice over IP (VoIP), video-on-demand, IP television (IPTV) technologies, high definition video conferences and some real-time applications, require mechanism and future research to meet QoS requirement. Multimedia applications require different QoS from simple network applications. Multimedia services need more bandwidth, lossless delivery, and strict delay. IP networks treat all traffic, in the same way, this will be a problem for real-time traffic likes multimedia applications. QoS is not a consideration at the beginning of the development of IP networks. However, as its growth and support more services including critical and real-time services, QoS support is indispensable.

Several studies discussed the way to provide QoS in the IP network. [2] uses MPLS as an automatic bandwidth allocation. [3] adopt MPLS technologies that provide high management capabilities and extreme flexibility and scalability, while supporting new applications with reasonable cost, high transmission rates, and predictable QoS [4]. Proposes a new per-class bandwidth constraint algorithm, called the multipath selection algorithm (MSA), for a Diff-Serv-TE network. This paper proposes a new per-class bandwidth constraint algorithm, called the multipath selection algorithm (MSA), for a Diff-Serv-TE network [3]. Use online allocation approach of communication and computation resources for real-time multimedia services. The most accepted and largely adopted solutions in computer network devices are IETF's IntServ [5] and DiffServ [6].

This paper presents a simulation analysis of Diffserv QoS management and MPLS network protocol to provide quality of service guarantees in a multimedia network. The aim of this study shows that two methods can improve network performance for multimedia type applications in massive traffic environments.

\section{LITERATURE REVIEW}

\subsection{Quality of Service}

Service on a network assumed to be an end-to-end service, or terminal (TE) to another terminal. End-to-end services are supported by mechanisms to determine the quality of service on a network. This mechanism is called Quality of Services (QoS). QoS defines the tools necessary to improve service quality [7].

\subsection{DiffServ}

Diffserv is an architecture to provide different types or service standards for network traffic. DiffServ was standardized in 1998 by IETF with a published request for comments (RFC) 2475. DiffServ guarantees QoS through two significant components: packet classification and per-hop behavior (PHB).

\footnotetext{
* Corresponding author: madesuartana@unesa.ac.id
} 


\subsubsection{Packet Classification}

Packet Classification on DiffServ through the different value of services code point (DSCP). DSCP consists of six data bits that replace the type of services (ToS) bytes in the header of a packet. The use of DSCP allows network devices to map service classes and provide different treatment.

\subsubsection{Per-hop Behavior (PHB)}

PHB defines the treatment given to a package based on the given classification. Types of PHB: Default PHB (Defined at RFC-2474), PHB Expedited Forwarding (Defined at RFC-2598), and PHB Assured Forwarding (Defined at RFC-2597).

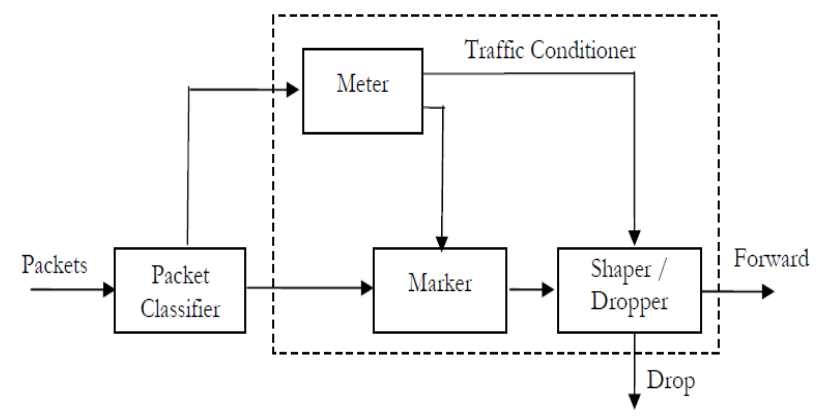

Fig. 1. DiffServ operation.

- Classifier: Selects a packet in a traffic stream based on the content of some portion of the packet header

- Meter: Checks compliance to traffic parameters (e.g., Token Bucket) and passes the result to the marker and shaper/dropper to trigger a particular action for in/out of profile packets

- Marker: Writes/rewrites DSCP

- Shaper: Delays some packets to be compliant with a profile

- Dropper: Discards some or all of the packets in a traffic stream to bring the stream into compliance with a traffic profile

\subsection{MPLS}

IntServ and DiffServ have a limitation in scalability and simplicity. To overcome these limitations, the Internet Engineering Task Force (IETF) established the MultiProtocol Label Switching (MPLS) Working Group and implemented MPLS technology standards. MPLS is a packet delivery technology on high-speed backbone networks. Its working principle combines some of the advantages of circuit-switched and packet-switched communication systems that give rise to better technology from both. Without MPLS, packets forwarded with routing protocols such as OSPF, IS-IS, BGP, or EGP. The routing protocol resides on the network layer in the OSI system, whereas MPLS is between the second and third layers.

\subsubsection{Core Technology MPLS Network}

MPLS's working principle is to combine the switching speed of layer 2 with the routing and scalability capabilities of layer 3 . The way it works is by inserting labels between the layer 2 and layer three headers in the forwarded packets. Labels are generated by the LabelSwitching Router which acts as an MPLS network connector with an outside network. The label contains the destination information of the next node in the packet delivery process. Then the packet is forwarded to the next node, at this node the packet label will be removed and labeled a new one containing the next destination. The LSP (Label Switching Path) is a path to transmitted packets.

\subsubsection{Core Technology MPLS Network}

Label Switched Path (LSP): A path that passes through one or a series of LSRs where packets are passed by a swapping label from one MPLS node to another MPLS node. Label Switching Router: MPLS node capable of forwarding layer-3 packets. MPLS Edge Node or Label Edge Router (LER): MPLS node that connects an MPLS domain with nodes that are outside the MPLS domain. MPLS Egress Node: MPLS node that controls traffic when leaving the MPLS domain. MPLS ingress Node: MPLS node that controls traffic when going into MPLS domain. MPLS label is a label placed as MPLS header. MPLS node: a node that runs MPLS, this node as a protocol control that will forward packets based on labels.

\section{Simulation approach}

This paper used the simulation approach to analysis purpose method. The simulation following steps as shown in figure 2.

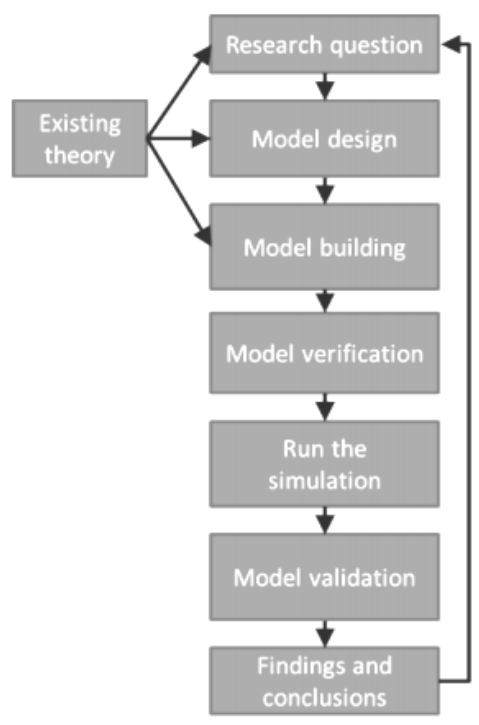

Fig. 2. Steps in a simulation [8].

To compare the quality of service guarantees using simulation. Model design, building and verification in 
simulation steps using Network Simulator-3(NS-3). NS3 is a discrete-event network simulator for Internet systems, targeted primarily for research and educational use [9]. This paper used NS-3.26 software version with Diffserv and MPLS Network Simulator.

The simulation design uses 9 IP nodes. All links between node utilizing the duplex link with $10 \mathrm{~ms}$ delay and Drop-Tail Queuing. This queuing base on a First Come First Serve (FCFS) algorithm that serves packet that came first come. Each topology data rate link has a bandwidth capacity $2 \mathrm{Mbps}$ except for the links from node three to node four has only 1 Mbps link capacities. Therefore, the connection from node three to node four expected to run into bottlenecks. The network simulation will use a real-time and best-effort traffic. Best-effort traffic as background traffic, to show priority to realtime traffic into QoS related interests. Real-time traffic connection used node 0 as a sender and node eight as a receiver, this traffic using UDP utilize CBR with packet size 512-byte and inter-arrival time packet $3 \mathrm{~ms}$. The best effort traffic connection used between node one and nine as a sender and receiver, using TCP with packet sizes of 512-bytes. The TCP source using FTP, and it sends packets to allow window congestion. To measure the performance of the application of QoS mechanism, the simulation output trace file used to measure the accomplishments of the network such as TCP and UDP throughput, delay, and packet loss.

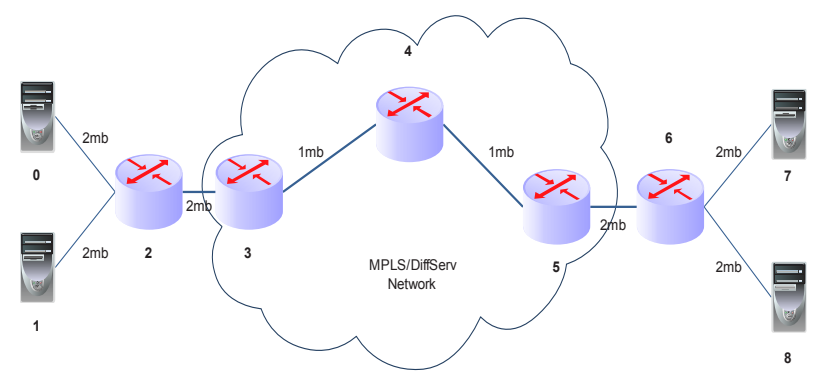

Fig. 3. Simulation topology.

The simulation topology describes in figure 3, both the TCP and UDP traffic use the same path. QoS mechanisms are expected to give priority to real-time traffic, which is in this simulation using UDP traffic.

This simulation only runs for about 5 seconds. the output of the simulation is analysed and compared between the Diffserv model, MPLS and best-effort service in IP network. Minimum requirements in accordance with ITU-G1010 must be satisfied to ensure QoS in multimedia network.

\section{Performance evaluation}

In this section, we present simulation results. Using DiffServ QoS management because this model is the current trend on the Internet for the development of a scalable QoS mechanism [1] and MPLS be able to ensure the end-to-end consistency of QoS [10].

Different standardization groups, like ITU, ETSI or 3GPP, have covered QoS requirements for multimedia traffic. ITU is in Recommendations G.1010 [11][12].
Applications have been classified into eight groups, according to the error tolerance and delay, as summarized in Figure 4.

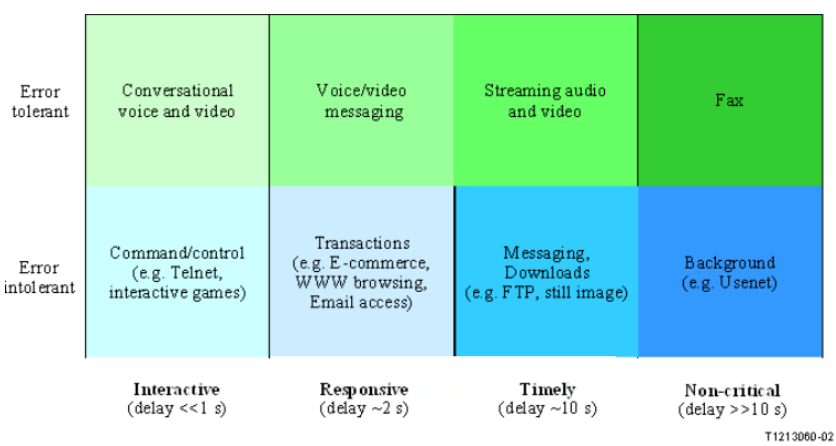

Fig. 4. G.1010 - Model for user-centric QoS categories.

Based on simulation results IP network without QoS mechanism only provides best effort service. When the TCP and UDP traffic uses the same paths to deliver data, causes congestion and overlap to this path. When the link of the network is busy, congestion is occurring within the network. Packets from connection node three to node four get dropped and delayed as buffers overflow because the resources in the network cannot meet all traffic demands.

Using QoS mechanism TCP flow reduction expected by mixing UDP and TCP traffic in the same line, when the UDP increase the flow rate. Both the MPLS or DiffServ shows the same result of increased throughput on UDP traffic. Comparison of simulation results using Diffserv on UDP and TCP traffic, which prioritizes realtime traffic in this case represented by UDP shown in table 1, in which TCP receivers only have 2484 packages, and UDP has a 3015-packet reception. Because of this simulation only runs for about 5 seconds, it means that UDP throughput is only slightly higher than the throughput of TCP. If the simulation was allowed to continue for a longer period, it is possible that the UDP throughput would overlap TCP throughput and packet loss would be much higher.

Table 1. DiffServ simulation result.

\begin{tabular}{|l|c|c|c|c|}
\hline & \multirow{2}{*}{ Delay(s) } & \multicolumn{2}{|c|}{ Packet loss } & Throughput \\
\cline { 3 - 4 } & & Sent & Loss & (Kbps) \\
\hline DiffServ & 0.0762663 & 3656 & 641 & 1521,75 \\
\hline Best-Effort & 0.0770471 & 3654 & 1170 & 820,51 \\
\hline
\end{tabular}

Table 2, show simulation results using MPLS on UDP and TCP traffic, TCP receivers only have 2487 packages, and UDP has a 2947 packet reception.

Table 2. MPLS simulation result.

\begin{tabular}{|c|c|c|c|c|}
\hline & \multirow{2}{*}{ Delay(s) } & \multicolumn{2}{|c|}{ Packet loss } & \multirow{2}{*}{$\begin{array}{c}\text { Throughput } \\
\text { (Kbps) }\end{array}$} \\
\hline & & Sent & Loss & \\
\hline MPLS & 0.0770432 & 3656 & 709 & 1420,8 \\
\hline Best-Effort & 0.0970471 & 3654 & 1167 & 816,58 \\
\hline
\end{tabular}




\section{Conclusion}

In this paper, we addressed the problem to analyze and compare the quality of service guarantees provided by Diffserv QoS model and Multi-Protocol Label Switching in a multimedia network, based on simulation approach. IP networks treat all traffic, in the same way, this will be a problem for real-time traffic likes multimedia applications. This project uses Multi-Protocol Label Switching and DiffServ QoS management schemes to meet the quality of service requirement in multimedia networks. Experimental results reveal that the proposed QoS method can improve the performance significantly compared to best-effort services that provide by IP Network.

\section{References}

1. H. Wu-Hsiao, T. Ming-Chih, W. Li-Yuan, Computer Communications 30, 1406-1418 (2007)

2. N. Akar, M. A. Toksoz, Computer Networks 55, 1181-1196 (2011)

3. L. Jun, A. Philip, Chou Chun Yuan, Yusuo Hu, Wenwu Zhu, IEEE Transactions on Multimedia 15, 3, $670(2013)$

4. H. Wu-Hsiao, S. Yuh-Pyng, C. Jenhui, Computer Communications 33, 1557-1565 (2010)

5. Babiarz, J, Chan. K, Nortel Networks, F. Baker, Cisco Systems (2006)

6. Y. Bernet, P. Ford, R. Yavatkar, F. Baker, L. Zhang, M. Speer, R. Braden, B. Davie, J. Wroclawski, E. Felstaine, The Internet Society (2000)

7. Technical Specification, 3GPP (2010)

8. J. P. Davis, K. M. Eisenhardt, C. B. Bingham, Academy of Management Review 32, 2, 480-499 (2007)

9. F. Pedro, R. Manuel, NSTOOLS (2009)

10. A. Hakiri, P. Berthoua, A. Gokhalec, D. C. Schmidt, G. Thierrya, JSS-9318 2-22 (2014)

11. ITU-T Recommendation G.1010 (2001)

12. Q. M. Qadir, A. A. Kist, Z. Zhang, IEEE Transactions on Multimedia 17, 5, 711-722 (2015) 